\title{
Muon transfer from hot muonic hydrogen atoms to neon
}

\author{
R. JACOT-GUILLARMOD
}

DE93 010250

Institut de Physique, Pérolles, CH-1700 Fribourg, Switzerland

J.M. BAILEY

University of Liverpool, P.O Box 147, Liverpool L69 $3 B X, U K$

G.A. BEER, P.E. KNOWLES, G.R. MASON and A. OLIN

University of Victoria, Finnerty Road, Victoria, B.C. V8W $2 Y^{\prime 2}$, Canada

J.L. BEVERIDGE and G.M. MARSHALL

TRIUMF, 4004 Wesbrook Mall, Vancouver, B.C. V6T 2A3, Canada

J.H. BREW.ER and B.M. FORSTER

University of British Columbia, 6224 Agricultural Road, Vancouver, B.C. V6T 2A6, Canada

T.M. HUBER

Gustavus Adolphus College, St. Peter, MN 56082, USA

P. KAMMEL and J. ZMESKAL

IMEP, Austrian Academy of Sciences, Boltzmanngasse S, A-1090 Wien, Austria

A.R. KUNSELMAN

University of Wyoming, Laramie, WY 82071, USA

C. PETITJEAN

Paul Scherrer Institute, CH-5232 Villigen, Switzerland

A negative muon beam has been directed on adjacent solid layers of hydrogen and neon. Three targets differing by their deuterium concentration were inves-

tigated. Muonic hydrogen atoms can drift to the neon layer where the muon is immediately transferred. The time structure of the muonic neon $\mathrm{X}$-rays follows the exponential law with a disappearance rate corresponding to the one of $\mu^{-} \mathrm{p}$ atoms in each target. The rates $\lambda_{\mathrm{pp} \mu}$ and $\lambda_{\mathrm{pd}}$ can be extracted.

\section{DISCLAIMER}

This report was prepared as an account of work sponsored by an agency of the United States Government. Neither the United States Government nor any agency thereof, nor any of their employees, makes any warranty, express or implied, or assumes any legal liability or responsibility for the accuracy, completeness, or usefulness of any information, apparatus, product, or process disclosed, or represents that its use would not infringe privately owned rights. Reference herein to any specific commercial product, process, or service by trade name, trademark, manufacturer, or otherwise does not necessarily constitute or imply its endorsement, recommendation, or favoring by the United States Government or any agency thereof. The views and opinions of authors expressed herein do not necessarily state or reflect those of the United States Government or any agency thereofi. 


\section{Introduction}

This study has historically grown as an extension of a TRIUMF experiment which is already described in this volume [1]. Thus we won't repeat here most of the features which are described there, but concentrate on those details which are specific to this contribution.

By slowing down via Coulomb interaction with electrons, muons are stopped in a thin solid layer of hydrogen containing a small amount of deuterium. In this dense material, the $\mu^{-} \mathrm{p}$ atoms are thermalized in a very short time $\left(\sim 10^{-12} \mathrm{~s}\right)$ compared to the $\mu^{-}$lifetime. Different $\mu^{-} \mathrm{p}$ disappearance channels exist : muon decay, $\mathrm{pp} \mu$ molecular formation or muon transfer to cieuterium and to impurities. After muon transfer from $\mu^{-} \mathrm{p}$, the muonic deuterium atom has an initial kinetic energy of about $45 \mathrm{eV}$. Most of them are slowed down by interacting on protons until they reach a few eV where the cross-section drops due to the Ramsauer-Townsend effect.

By using a decay $\mathrm{e}^{-}$imaging system, the emission of these "hot" $\mu^{-\mathrm{d}}$ in vacuum has been extensively studied in terms of deuterium concentration dependence and layer thickness [1]. Because of their high velocity $(\sim 1 \mathrm{~cm} / \mu \mathrm{s})$, these $\mu^{-} \mathrm{d}$ have time to drift a few centimeters away from the hydrogen layer before muon decay occurs. Therefore the decay $\mathrm{e}^{-}$imaging system was a very useful device for observing them. Although their velocity should be very slow compared to these $\mu^{-\mathrm{d}}(\sim 0.1 \mathrm{~mm} / \mu \mathrm{s})$, the emission of residual $\mu^{-}$p atoms can not be completely excluded. The decay electron imaging system is, however, unable to extract their contribution from the target background. 
The aim of this work is to study individually the $\mu^{-} \mathrm{p}$ and $\mu^{-} \mathrm{d}$ emission from the hydrogen layer. The basic idea is to freeze a thin neon layer on the surface of hydrogen. If muonic hydrogen atoms are emitted in this direction, these will instantaneously release their muon to form excited muonic neon atoms by transfer. Characteristic $\mathrm{X}$-rays will be emitted during the cascade and observed with a $\mathrm{Ge}$ intrinsic semiconductor detector.

If one neglects the $\mu^{-} \mathrm{p}$ and $\mu^{-} \mathrm{d}$ drift time inside the hydrogen layer (this process remains to be studied in detail), the time distribution of the muonic neon $\mathrm{X}$-rays emitted after muon transfer should have an exponential shape with an associated rate $\lambda$ corresponding to the disappearance rate of $\mu^{-} \mathrm{p}$ atoms inside the hydrogen layer:

$$
\lambda=\lambda_{0}+\left(1-c_{d}\right) \lambda_{\mathrm{pp} \mu}+c_{d} \lambda_{p d}
$$

where the transfer to impurities has been neglected. The $\mathrm{X}$-rays emitted after direct muon stop in the neon layer will be observed promptly and can be easily separated from the other events.

\section{Measurement}

The apparatus set-up is shown on Fig. 1. To be as careful as possible with gas mixing, and to get a maximum of information from our detectors set-up, we proceeded in two steps. The hydrogen layer was first frozen on the gold foil and the $\mu^{-\mathrm{d}}$ emission observed using the imaging system. Simultaneously, the X-rays were measured to 
identify the presence of impurities if any. Then, some neon was frozen on the hydrogen layer surface without warming excessively the target, and the muonic neon $\mathrm{X}$-rays were collected.

This technique was applied for three targets which differed by their thickness and deuterium concentration as shown in Table 1.

\section{Data analysis}

The full data evaluation is not yet complete, the values reported here are also preliminary. Fig. 2 shows typical total energy spectra obtained with natural isotopic concentration of $\mathrm{D}_{2}$ in hydrogen (target II), before (2a) and after (2b) the neon layer deposition. The absence of low $\mathrm{Z}$ element $\mathrm{X}$-rays in Fig. 2a, and especially those from neon, indicates clearly that the impurity contamination in the hydrogen is negligible. Most of the neon line intensity of Fig. $2 \mathrm{~b}$ comes from direct $\mu^{-}$stops in the neon layer.

The specific contribution of the transfer process from muonic hydrogen can be observed by requiring a delayed time cut, as shown in Fig. 3 for the same data. It is essential to observe that the huge $\mu \mathrm{Au}(6-5)$ peak of Fig. 2 is completely absent in these delayed X-ray spectra. Therefore one can conclude that the energy-time correlation and the time resolution were satisfactory.

The complete absence of the delayed muonic gold $\mathrm{X}$-rays is a priori very intriguing be use one would expect, due to the isotropic $\mu^{-\mathrm{d}}$ emission, that delayed muonic gold X-rays would be apparent. In addition, no muonic carbon X-rays are observed. 
This allows us to rule out the presence of a thin carbon layer at the surface of the gold foil, as discussed for an analogous experiment in Ref. 2. The intensity of the muonic transition $\mu \mathrm{Au}(6-5)$ by direct $\mu^{-}$stop is about twenty times more than via $\mu^{-}$transfer, because the circular transitions are much more favoured for the former.

One can then assert that the delayed muonic neon X-rays of Fig. 3 follow the emission of muonic hydrogen into the adjacent neon layer where muon transfer eventually occurs.

Unfortunately, the statistics of the delayed events in the neon X-rays (and especially the 2-1), and the low signal to background ratio are insufficient to get a time spectrum of these events. We have proceeded by grouping the counts in four time bins: from 40 to $120 \mathrm{~ns}$ after $\mu^{-}$signal (this spectrum will be called Del I), from 121 to $339 \mathrm{~ns}$ (Del II), from 340 to 1539 ns (Del III) and from 1540 to 7931 ns (Del IV). The fitted intensities of $\mu \mathrm{Ne}(2-1)$ in each time bin are reported in Table 2.

The width of each time bin was chosen to have almost the same statistics in each. As previously stated, the delayed time structure of the neon $\mathrm{X}$-rays should be exponential. This statement was confirmed by fitting the intensity structure as a function of the time window. The lifetimes corresponding to each target are also reported in Table 2 .

Using Eq. 1, one obtains a set of three linear equations, each one corresponding to one value of deuterium concentration $c_{d}$, with two free parameters $\lambda_{p p \mu}$ and $\lambda_{p d}$. One finally gets : 


$$
\begin{aligned}
& \lambda_{\mathrm{pp} \mu}=(2.18 \pm 0.32) \cdot 10^{6} s^{-1} \\
& \lambda_{\mathrm{pd}}=(0.98 \pm 0.19) \cdot 10^{10} \mathrm{~s}^{-1}
\end{aligned}
$$

These values are in very good agreement with those obtained at comparable experimental conditions i.e. with liquid hydrogen in the sixties $[3-5]$. The rate $\lambda_{\text {pd }}$ however differs from the value which is generally accepted and measured at room temperature [6] of $(1.68 \pm 0.26) \cdot 10^{10} s^{-1}$, although recent calculations [7] do not predict such energy dependence.

\section{Acknowledgements}

The support of the Natural Sciences and Engineering Research Council (NSERC) of Canada is gratefully acknowledged. One of us (R. J.-G.) wishes to thank the Swiss National Science Foundation for his support. 


\section{References}

[1] See G.M. Marshall et al. contribution in this volume.

[2] J.B. Kraiman, W.H. Breunlich, M. Cargnelli, G. Chen, P.P. Guss, F.J. Hartmann, P. Kammel, J. Marton, J.J. Reidy, R.T. Siegel, W.F. Vulcan, R.E. Welsh, H. Woolverton, A. Zehnder, and J. Zmeskal, Phys. Fer. Lett. 63, 1942 (1989).

[3] V.P. Dzhelepov, P.F. Ermolov, E.A. Kushnirenko, V.I. Moskalev, and S.S. Gershtein, Sov. Phys. JETP 15, 306 (1962).

[4] E.J. Bleser, E.W. Anderson, L.M. Lederman, S.L. Meyer, J.L. Rosen, J.E. Rothberg, and I-T. Wang, Phys. Rev. 132, 2679 (1963).

[5] G. Conforto, C. Rubbia, E. Zavattini, and S. Focardi, Il Nuovo Cim. 33, 4281 (1964).

[6] A. Bertin, M. Bruno, A. Vitale, A. Placci, and E. Zavattini, Phys. Rev. A7, 462 (1973).

[7] C. Chiccoli, V.I. Korobov, V.S. Melezhik, P. Pasini, L.I. Ponomarev, and J. Wosniak, Muon Cat. Fusion 7, 87 (1992). 
Table 1: Composition of the three targets used. The $\oplus$ sign indicates that the material was added to the surface and not mixed. 1000 torr $l l \mathrm{H}_{2}$ is $4.2 \mathrm{mg} \cdot \mathrm{cm}^{-2}$.

\begin{tabular}{cccc}
\hline Target \# & Composition \\
\hline I. & 1506 torr $\cdot l \mathrm{H}_{2} \oplus 194$ torr $\cdot l \mathrm{Ne}$ \\
II. & 1454 torr $\left.\cdot l\left(\mathrm{H}_{2}+145 \mathrm{ppm} \mathrm{D}\right)_{2}\right)$ & 193 torr $\cdot l \mathrm{Ne}$ \\
III. & 725 torr $l\left(\mathrm{H}_{2}+1150 \mathrm{ppm} \mathrm{D} \mathrm{D}_{2}\right) \oplus$ & $\oplus 5$ torr $\cdot l \mathrm{Ne}$
\end{tabular}

Table 2: Intensity of the $\mu \mathrm{Ne}(2-1)$ line measured for different time windows. By assuming an exponential dependence of the delayed intensities, the lifetime $\tau=1 / \lambda$ of the $\mu^{-} \mathrm{p}$ is deduced.

\begin{tabular}{cccccccc}
\hline Target & $\mathrm{c}_{\mathrm{d}}$ & \multicolumn{3}{c}{$\mu \mathrm{Ne}(2-1)$} & intensity for each time cut & Lifetime \\
& $(\mathrm{ppm})$ & Prompt & Del I & Del II & Del III & Del IV & $\tau(\mathrm{ns})$ \\
\hline I & 0 & 9116 & 170 & 260 & 246 & 33 & 303 \\
& & $(125)$ & $(42)$ & $(37)$ & $(33)$ & $(33)$ & $(40)$ \\
II & \multirow{2}{*}{145} & 5032 & 228 & 311 & 171 & 27 & 210 \\
& & $(91)$ & $(34)$ & $(32)$ & $(26)$ & $(24)$ & $(21)$ \\
III & \multirow{2}{*}{1150} & 7057 & 365 & 110 & 20 & & 56 \\
& & $(100)$ & $(36)$ & $(28)$ & $(23)$ & & $(9)$
\end{tabular}


Figure 1: Experimental apparatus

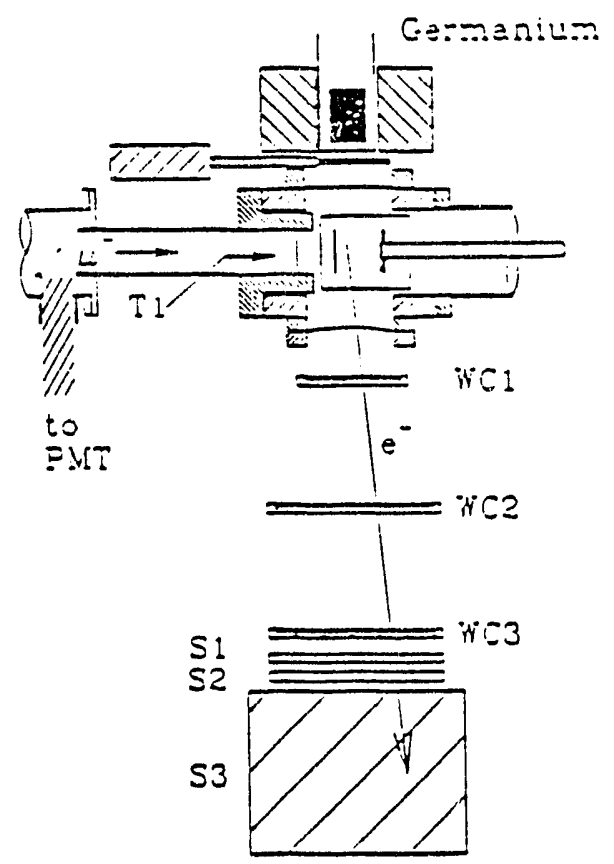


Figure 2: Part of the X-ray energy spectra without any time cut measured with target II (cf. Table 1). Upper (a) and lower (b) spectra have been obtained before and after neon deposition, respectively.

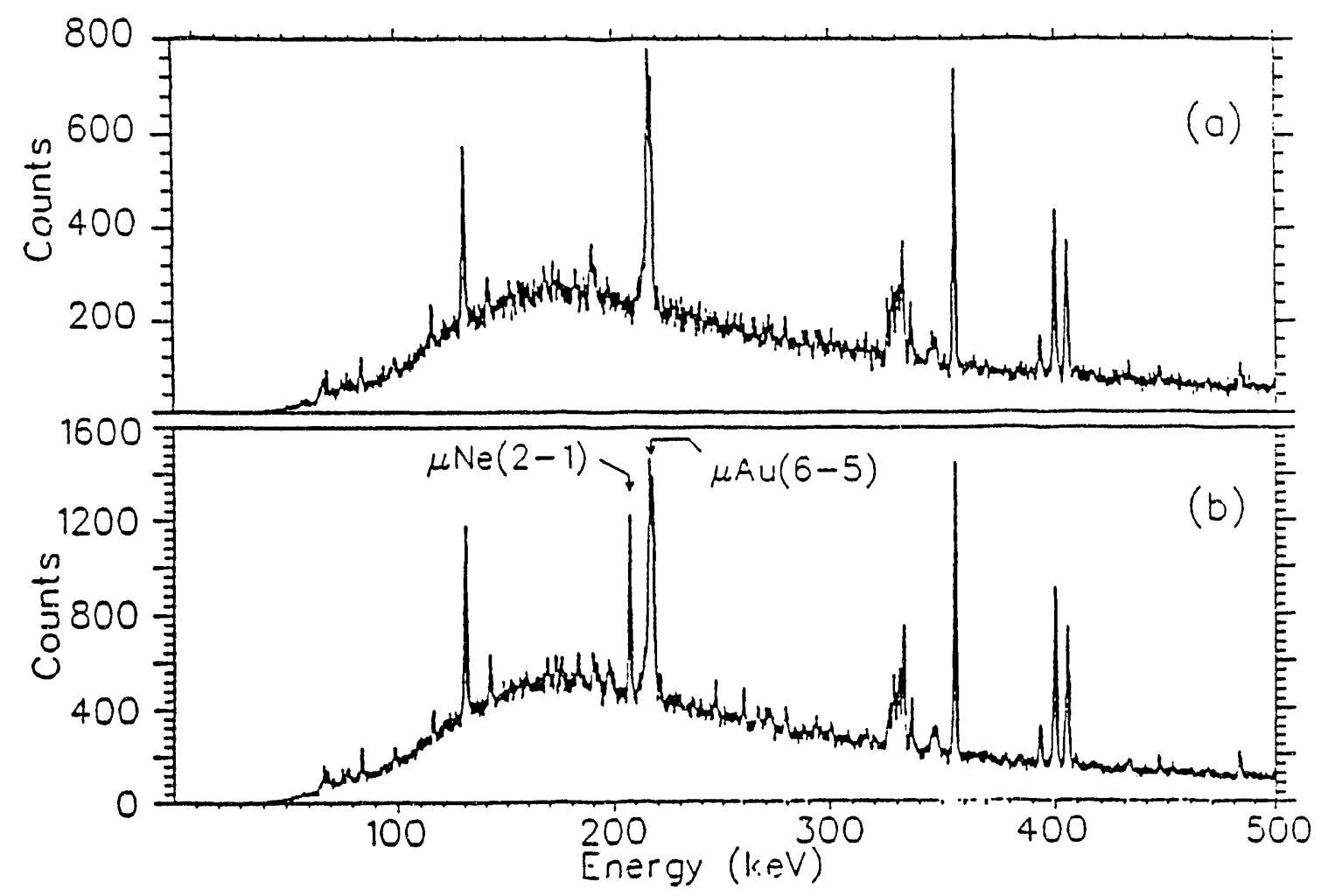


Figure 3: Part of the delayed X-ray energy spectra measured with target II (cf. Table 1). Upper (a) and lower (b) spectra have been obtained before and after neon deposition, respectively.

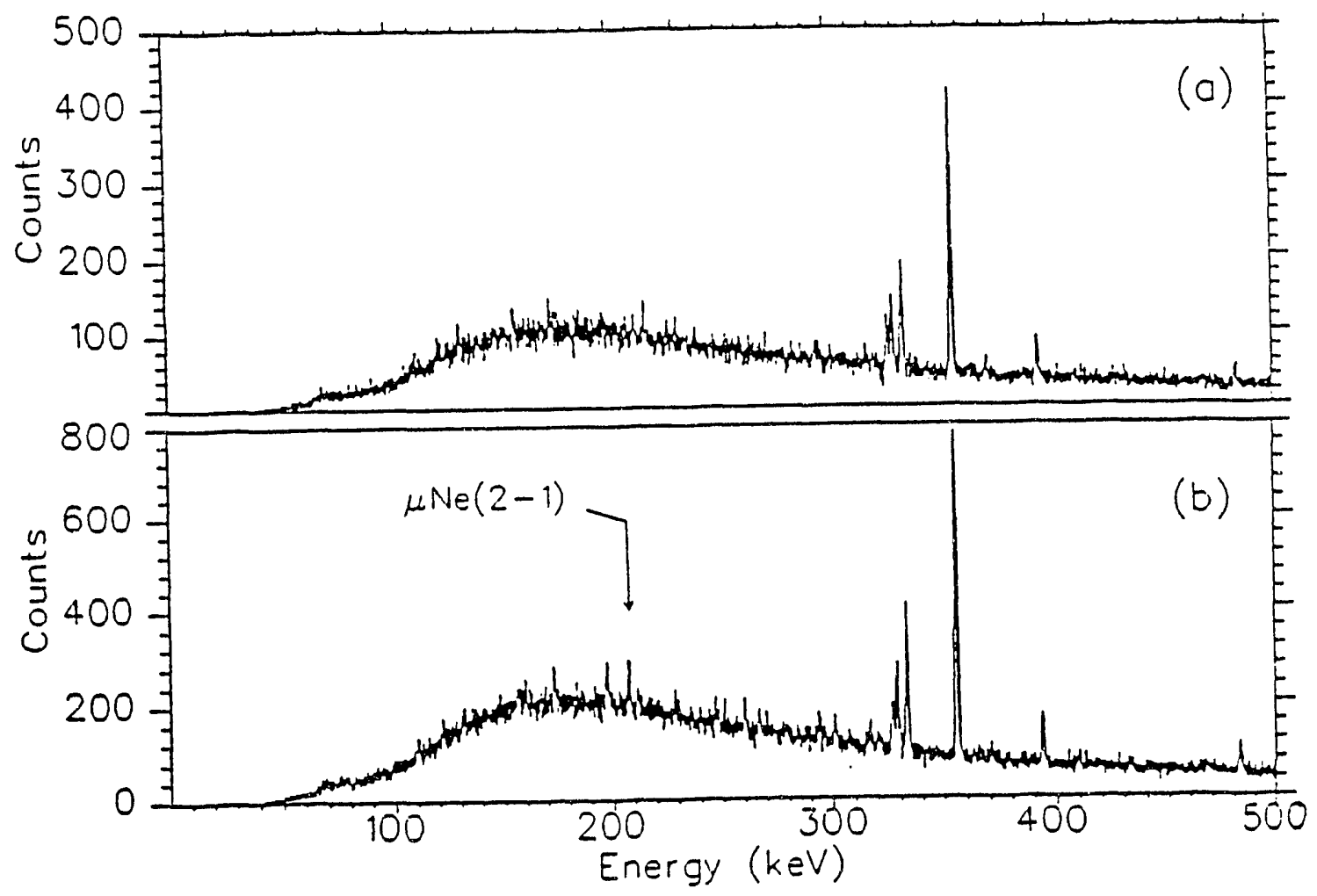



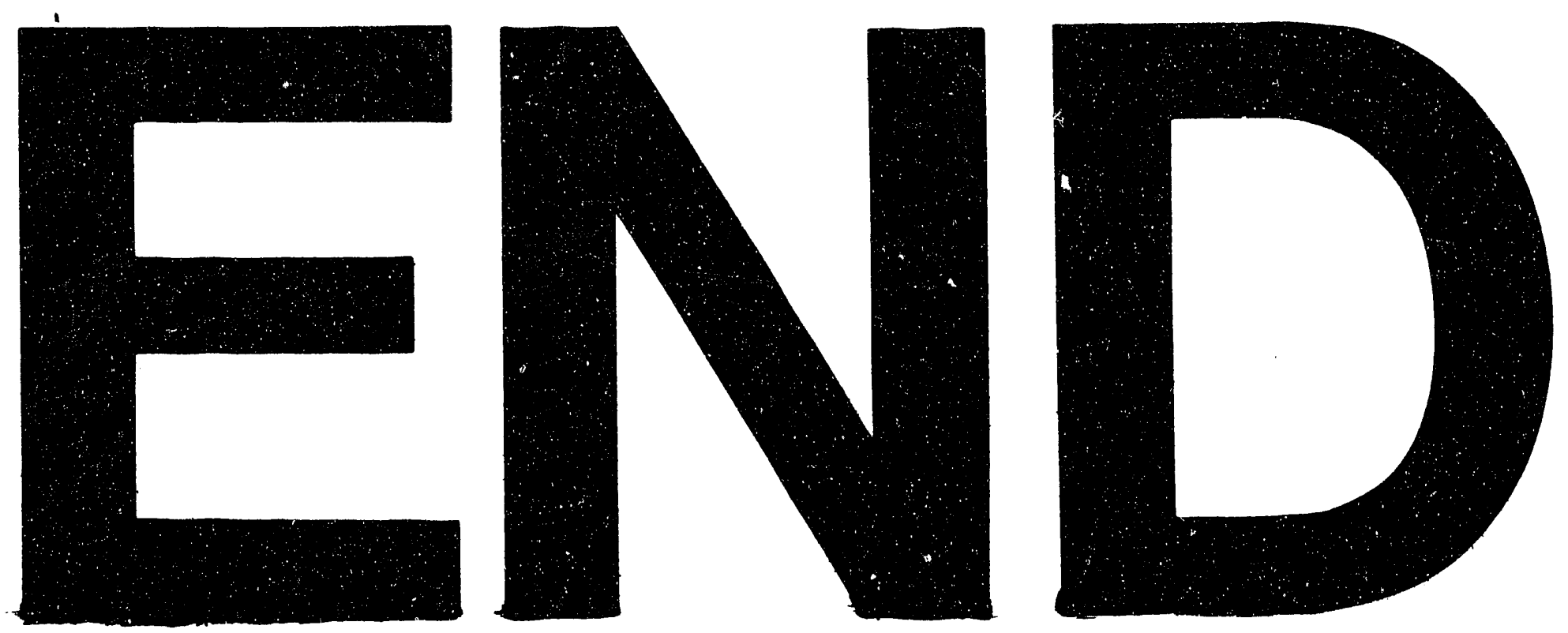

a

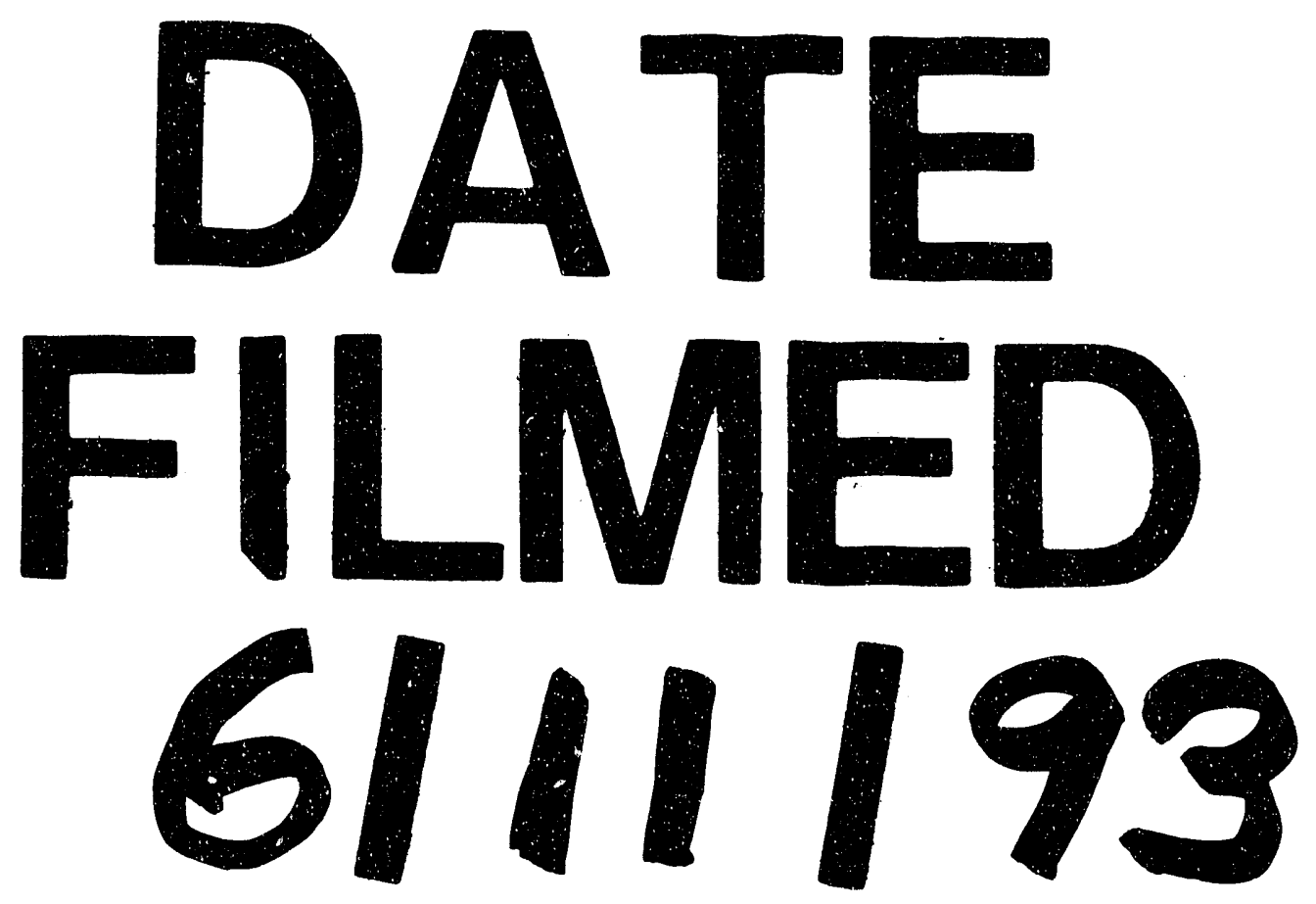

